\title{
Suppression of gastric cancer by extract from the tuber of amorphophallus konjac via induction of apoptosis and autophagy
}

\author{
XI CHEN $^{1,2}$, LIN-QING YUAN ${ }^{1}$, LIN-JIE LI $^{1}$, YAO LV ${ }^{1}$, PEI-FENG CHEN ${ }^{3}$ and LEI PAN ${ }^{3}$ \\ ${ }^{1}$ Children's Hospital, Zhejiang University School of Medicine, Hangzhou, Zhejiang; \\ ${ }^{2}$ Key Laboratory of Diagnosis and Treatment of Neonatal Diseases of Zhejiang Province, Hangzhou, Zhejiang; \\ ${ }^{3}$ The First Affiliated Hospital of Zhejiang Chinese Medical University, Hangzhou, Zhejiang, P.R. China
}

Received January 13, 2017; Accepted June 8, 2017

DOI: $10.3892 / o r .2017 .5747$

\begin{abstract}
The tuber of amorphophallus konjac (TuAK) is an antitumor herb used in traditional Chinese medicine. The present study investigated the inhibitory effect of TuAK against gastric cancer and the underlying mechanisms associated with two programmed cell death pathways, apoptosis and autophagy. TuAK was extracted by organic solvents including ethanol and ligarine. The extract of TuAK, shortened as TuAKe, significantly inhibited the growth of cultured gastric cancer cell lines SGC-7901 and AGS, with $\mathrm{IC}_{50}$ of 35-45 $\mu \mathrm{g} / \mathrm{ml}$. TuAKe could increase cell apoptosis and induce cell cycle arrest. For the apoptosis-associated proteins, expressions of survivin and $\mathrm{Bcl}-2$ were decreased by treatment of TuAKe, and the expression of Bax and caspase-9 was increased. Furthermore, TuAKe could promote autophagy, and the antitumor efficacy of TuAKe was significantly hampered by targeted suppression of autophagy, suggesting that autophagy contributed to TuAKe-induced cell death. Furthermore, patients with gastric cancer who received TuAK-based medicinal decoction achieved improved scores in assessment of life quality compared with those without TuAK treatment. This study demonstrated the antitumor activity of TuAKe against gastric cancer, and is the first report to show that the underlying mechanism is associated with induction of autophagy. Our data provided support of the clinical use of amorphophallus konjac-based medication in combination with classical chemotherapy to achieve optimized outcome for gastric cancer.
\end{abstract}

Correspondence to: Dr Lei Pan, The First Affiliated Hospital of Zhejiang Chinese Medical University, Hangzhou, Zhejiang 310006, P.R. China

E-mail: panlei21316@163.com

Dr Xi Chen, Children's Hospital, Zhejiang University School of Medicine, 3333 Bin Sheng Road, Bin Jiang, Hangzhou, Zhejiang 310052, P.R. China

E-mail: chchenxi@zju.edu.cn

Key words: amorphophallus konjac, gastric cancer, apoptosis, autophagy

\section{Introduction}

Natural compounds from medicinal herbs compose a significant part of first-line antitumor drugs, such as vinca alkaloids, texans, podophyllotoxin and camptothecins (vincristine, camptothecin and paclitaxel) (1). Since malignant tumors tend to develop chemoresistance, there is high demand for novel antitumor compounds in the clinical practice. Natural herbal medicine has become a promising source for novel discoveries, such as flavonoids (quercetin, silibinin) $(2,3)$, polyphenols (curcumin) (4), alkaloids (berberine) (5), as well as medicinal decoctions containing undefined bioactive ingredients (6).

There are approximately 170 species of amorphophallus worldwide (7), mainly distributed in tropical and subtropical Asia and are used as a food source as well as in traditional Chinese medicine (TCM). The tuber part from a major species of amorphophallus, amorphophallus konjac, locally produced in Zhejiang, China, has been used in the clinical practice of TCM against cancer for decades (8). Reports on the antitumor efficacy of tuber of amorphophallus konjac (hereafter referred to as TuAK) can be dated back to the 1990s (9). Recently, an Iranian group has also reported the antitumor effect of amorphophallus konjac tuber using human hepatoma $(10,11)$ and colon cancer (12) models. Thus, TuAK has become an acknowledged source of natural antitumor compounds.

Many antitumor compounds affect apoptosis and autophagy pathways, two major programmed cell death mechanisms (13). Autophagy is featured by delivery of intracellular contents to the lysosome for degradation. On one hand, it serves as an alternative metabolic pathway to provide extra nutrition and energy for cell survival; on the other hand, over-activation of autophagy results in cell death by self-digestion, which has been defined as type II programmed cell death (14). Autophagy-targeting drugs such as chloroquine (CQ) have been applied in clinical trials for highly malignant and resistant tumors (www.clinicaltrials.gov). Thus, autophagy activation is a double-edged sword in tumorigenesis and antitumor therapy, facilitating either cell survival or cell death, depending on the cellular context (15). Some TCM herbs have been found to regulate the level of autophagy (16-18). However, whether they are beneficial or detrimental for the tumor cells is context-dependent and requires detailed investigation under different conditions. 
Our group has been investigating the efficacy of TuAK as well as the underlying mechanism for over 10 years in both clinical and laboratory circumstances (19). Recently, we reported the suppression of hepatocellular carcinoma by TuAK extracts both in vitro and in vivo via induction of apoptosis through survivin and Bax (20). In this study, the inhibitory effect of organic solvent-based TuAK extract (shortened as TuAKe) against gastric cancer cells was studied, as well as the underlying mechanisms associated with induction of cell cycle arrest, apoptosis and autophagy. We also report data on a retrospective study on life quality assessment in gastric cancer patients who received TuAK-based decoction while undergoing conventional chemotherapy.

\section{Materials and methods}

Reagents and antibodies. TuAK was purchased from Zhejiang Lin-An Medicinal Herbs, Co., Ltd., (Zhejiang, China; Batch no. 061208) and was authenticated by Zhejiang Chinese Medical University. A voucher specimen is maintained in the university. 5-Fluorouracil (5-FU) was from Nantong Jinghua Pharmaceutical Group, Co., Ltd., (Nantong, China). Chloroquine (CQ) was purchased from Sigma-Aldrich (St. Louis, MO, USA). Other chemical reagents (grade AR) were from Sigma-Aldrich, Amresco (Solon, OH, USA) or Aladdin Reagents Co., Ltd. (Shanghai, China). The following antibodies were used: survivin (ZSGB-Bio, Beijing, China), Bax (ZSGB-Bio), Bcl-2 (Santa Cruz Biotechnology, Santa Cruz, CA, USA), caspase-9 (Abcam, Cambridge, MA, USA), LC3 (Sigma-Aldrich), Atg7 (Cell Signaling Technology, Danvers, MA, USA) $\beta$-actin (Lianke Bio, Hangzhou, China) and GAPDH (Lianke Bio). The secondary antibodies were conjugated to HRP (Jackson Immunolab, West Grove, PA, USA).

Preparation of TuAK extracts. Dried TuAK was ground to fine particles, soaked in 8x volume of $95 \%$ ethanol overnight, and extracted twice by reverse flow, $2 \mathrm{~h}$ each time. The ethanol collected was further extracted by $2 \mathrm{x}$ volume of ligarine twice. The ligarine extract was recovered and concentrated by distillation in reduced pressure. The concentrated extract was filtered to obtain the organic solvent-based extract. The ethanol residuum was extracted by $\mathrm{dH}_{2} \mathrm{O}$ twice, $10 \mathrm{x}$ and $8 \mathrm{x}$ volume respectively, and concentrated to obtain the aqueous extract of TuAK. One mega-extraction using $15 \mathrm{~kg}$ of TuAK was performed and the extracts obtained were stored at $4^{\circ} \mathrm{C}$ with desiccants for the following experiments. The organic solvent-based extract of TuAK is hereafter referred to as TuAKe.

Cell culture and cell viability assay. SGC-7901, AGS and HEK293 cells were purchased from the Institute of Biochemistry and Cell Biology, Chinese Academy of Science (Shanghai, China). Cells were cultured in Dulbecco's modified Eagle's medium (DMEM; Gibco, Carlsbad, CA, USA) containing $10 \%$ fetal bovine serum (FBS; Gibco) at $37^{\circ} \mathrm{C}$ with $5 \% \mathrm{CO}_{2}$. For the viability assays, cells were seeded in 96-well plates, $1 \times 10^{4}$ cells/well. Desired drugs, compounds and extracts were added to the wells immediately afterwards and incubated with the cells for $24 \mathrm{~h}$. The cell viability was measured by spectrometry (Multiscan MK3 spectrophotometer; Thermo Fisher Scientific, Inc., Waltham, MA, USA) using CCK-8 kit (Lianke Bio) at OD $450 \mathrm{~nm}$.

Flow cytometric (FCM) analysis. Cells were seeded in 6-well culture plates and treated by desired drugs or extracts, or by phosphate-buffered saline (PBS) as control for $24 \mathrm{~h}$, then trypsinized and washed by PBS. Aliquots of resuspended cells $\left(5 \times 10^{5} / \mathrm{ml}\right)$ were stained by cell cycle and apoptosis analysis kit (Beckman Coulter, Brea, CA, USA) based on the manufacturer's instructions. FCM analysis was performed immediately afterwards (FACSAria ${ }^{\mathrm{TM}}$; BD Biosciences, San Jose, CA, USA).

Immunofluorescence. Cells were seeded in 24-well culture plates on glass coverslides, and treated at the desired conditions, then fixed and observed under a fluorescence microscope (IX71-22FL/PH; Olympus, Tokyo, Japan, or Axio A1; Carl Zeiss AG, Oberkochen, Germany).

Western blotting. Cells after desired treatment were scraped, spun down, washed by PBS and resuspended in RIPA buffer with standard protease and phosphatase inhibitors. After quantification of protein concentration by BCA method (Lianke Bio), cell lysates were mixed with loading buffer and subjected to SDS-PAGE with $20 \mu \mathrm{g}$ protein per lane. The proteins were later transferred to PVDF membranes, which were incubated by primary and secondary antibodies. The membranes were developed with an enhanced chemiluminescence kit (Lianke Bio). Each experimental condition was repeated for at least three times. Quantitative analysis of protein levels determined by band intensities was carried out by ImageJ (v1.48u).

Quantitative reverse transcription polymerase chain reaction $(q R T-P C R)$. Total RNA was extracted by TRIzol ${ }^{\circledR}$ reagent (Invitrogen, Carlsbad, CA, USA) and reverse transcripted to cDNA by a commercial kit (Takara Bio, Shiga, Japan). Primers for $\beta$-actin, $B C L 2$ and $B A X$ were synthesized by Invitrogen (Shanghai, China) with the following sequences: $\beta$-actin forward, (5'-ACAACTTTGGTATCGTGGAAGGAC-3') and $\beta$-actin reverse, (5'-AGGTGGAGGAGTGGGTGTCG-3'), $B C L 2$ forward, (5'-ATTGGGAAGTTTCAAATCAGC-3'), $B C L 2$ reverse, (5'-TGCATTCTTGGACGAGGG-3'), BAX forward, (5'-GACACCTGAGCTGACCTTGG-3') and $B A X$ reverse, (5'-GAGGAAGTCCAGTGTCCAGC-3'). PCR was performed in three independent experiments with multiple repeats (Applied Biosystems StepOnePlus; Applied Biosystems, Foster City, CA, USA) and the products were analyzed by agarose electrophoresis to compare mRNA levels.

Electron microscopy $(E M)$. Cells after treatment were scraped in PBS and spun down. The pellets were fixed by $2.5 \%$ glutaraldehyde for one week, then washed by $0.1 \mathrm{M}$ PBS and fixed in $1 \%$ osmic acid for $1 \mathrm{~h}$, washed by $\mathrm{ddH}_{2} \mathrm{O}$, dehydrated by serial concentrations of ethanol from 50 to $100 \%$. Then the pellets were dehydrated by $100 \%$ acetone, permeated by acetone/resin $(1: 1, \mathrm{v} / \mathrm{v})$ for $2 \mathrm{~h}$ and by pure resin overnight. The resin was polymerized by serial heating at 37,45 and $60^{\circ} \mathrm{C}$, sectioned and stained by uranium acetate-lead citrate, and then observed 

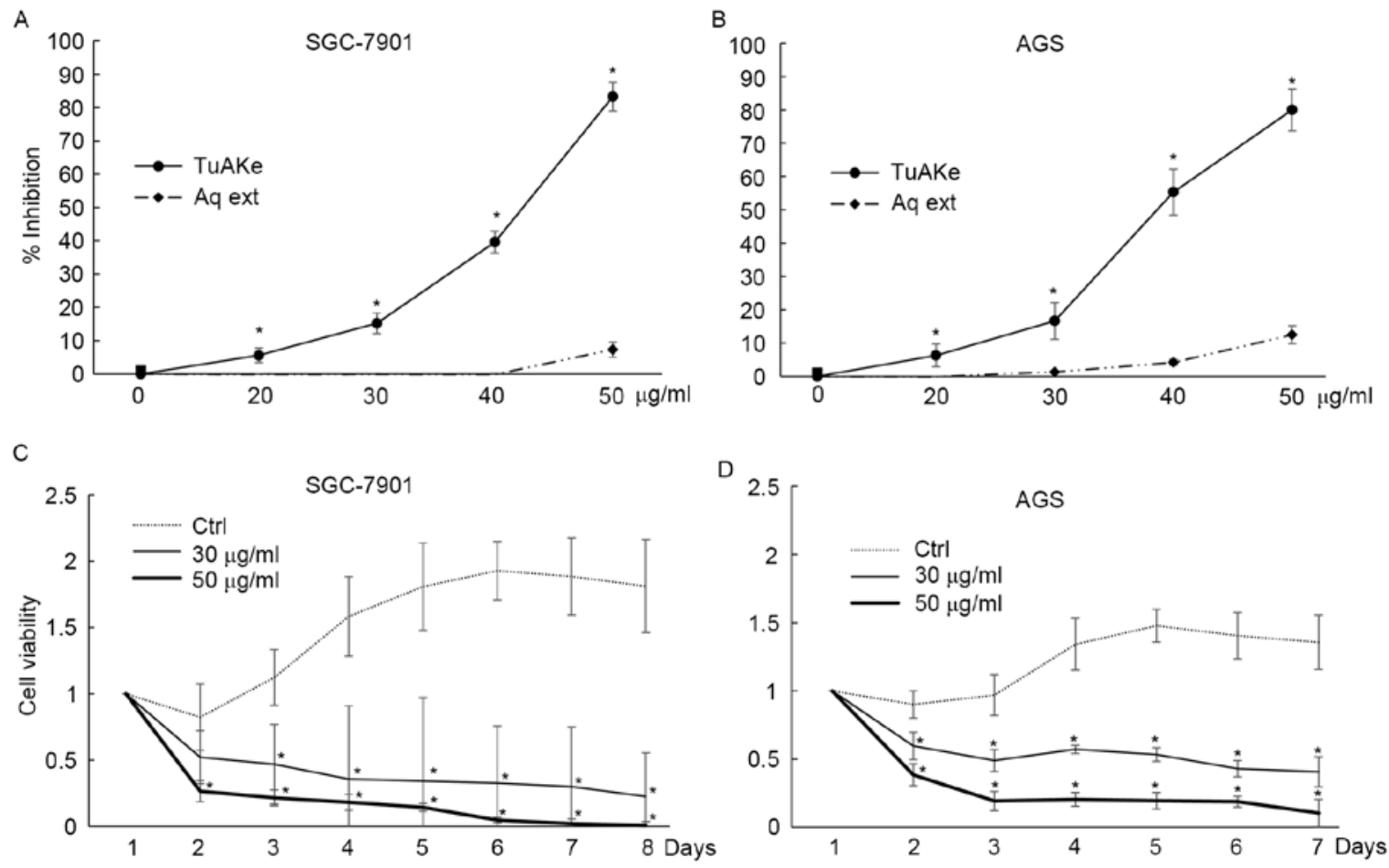

Figure 1. The antitumor activity of the tuber of amorphophallus konjac (TuAK) on gastric cancer cell lines. (A and B) The organic solvent-based extract of TuAK, TuAKe and the aqueous extract from the residuum (Aq ext) were incubated with cultured gastric cancer cell lines, SGC-7901 and AGS, for $24 \mathrm{~h}$. The inhibition rates were calculated after cell viability assays and are presented as mean $\pm \mathrm{SE}(\mathrm{n}=4)$. ${ }^{*} \mathrm{P}<0.05$, compared with the aqueous extract. (C and $\left.\mathrm{D}\right) \mathrm{TuAKe}$ at 30 or $50 \mu \mathrm{g} / \mathrm{ml}$ was incubated with cultured SGC-7901 and AGS cells for 7-8 days, with desired doses added fresh daily. PBS was used as the blank control. The inhibition rates were calculated based on cell viability assays, and were presented as mean $\pm \mathrm{SE}(\mathrm{n}=3)$. ${ }^{\mathrm{P}} \mathrm{P}<0.05$, compared with controls.

by EM equipped by the companion software (Tecnai; Phillips, Amsterdam, the Netherlands).

Knocking-down the autophagy-related gene ATG7. Cultured cells were infected with lentivirus expressing shRNA targeting ATG7 gene and control scrambled shRNA (Novobio, Shanghai, China) in the presence of polybrene. Twenty-four hours after infection, knocking-down of Atg7 was confirmed by western blotting, and cells were treated by TuAK for another $24 \mathrm{~h}$, with or without CQ for the last $6 \mathrm{~h}$, followed by cell viability assay.

Retrospective cohort study of gastric cancer patients treated by TuAK-based decoction. The institutional ethics committee of Zhejiang Chinese Medical University approved this study and waived the necessity to obtain informed consent based on its nature as a retrospective and observational study. A total of 30 inward gastric cancer patients admitted to the First Affiliated Hospital of Zhejiang Chinese Medical University were grouped into two. Ten patients in the control group received conventional chemotherapy using FAM regimen, namely combination of 5-FU, adriamycin and mitomycin $\mathrm{C}$. Twenty patients in the TuAK group received TuAK-based decoction in addition to the FAM regimen. The evaluated Karnofsky performance status (KPS) scores of all patients were over 60 upon admission to the hospital, and showed no statistically signifcant difference between the two groups. KPS scores of each patient were re-evaluated after 8 weeks of treatment and compared.

Statistical analysis. Each experiment was performed at least three times with multiple repeats, and the data were analyzed by SPSS (v13.0). The statistical significance was calculated by one-way ANOVA to compare between multiple groups, or by Student's t-test when only two groups were compared. $\mathrm{P}<0.05$ was considered statistically significant.

\section{Results}

The in vitro antitumor activity of TuAK extracts. In cultured human gastric cancer cell lines SGC-7901 and AGS, within $24 \mathrm{~h}$ of treatment, the inhibitory effect of the aqueous extract of TuAK was trivial compared with the organic extract, TuAKe (Fig. 1A and B). Based on the inhibition curves, in the two cell lines, the $\mathrm{IC}_{50}$ of TuAKe was $35-45 \mu \mathrm{g} / \mathrm{ml}$, while the aqueous extract had little inhibitory effect. TuAKe, the more potent extract, was used in the following experiments. In long-term experiments, consecutive treatment of $50 \mu \mathrm{g} / \mathrm{ml}$ TuAKe daily killed $\sim 100 \%$ SGC-7901 cells and $\sim 80 \%$ of AGS cells within 7 days, while lower dose of TuAKe $(30 \mu \mathrm{g} / \mathrm{ml})$ inhibited $\sim 70 \%$ of SGC-7901 cells and 50\% of AGS cells (Fig. 1C and D).

TuAKe induces cell apoptosis and cell cycle arrest. We analyzed the apoptotic rate in TuAKe-treated SGC-7901 cells. In Fig. 2A, FCM was carried out to detect the apoptotic cells induced by TuAKe after co-staining of Annexin V-FITC and PI. After treatment of $24 \mathrm{~h}, 5-\mathrm{FU}$ induced significant increase of apoptosis; $25 \mu \mathrm{g} / \mathrm{ml} \mathrm{TuAKe} \mathrm{increased} \mathrm{the} \mathrm{apoptotic} \mathrm{rate} \mathrm{but}$ without statistical significance. After cells were treated by 50 or $100 \mu \mathrm{g} / \mathrm{ml} \mathrm{TuAKe}$, cell apoptosis rates were significantly increased (13.3 and $11.2 \%$, respectively) to similar level as the 5-FU-treated group (12.7\%). 


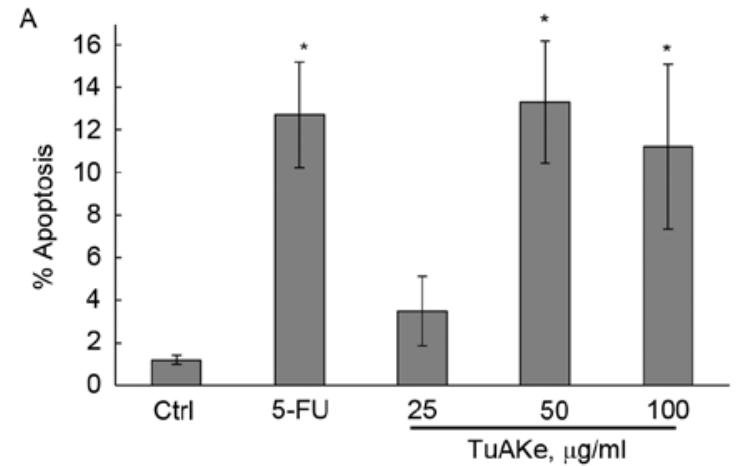

C

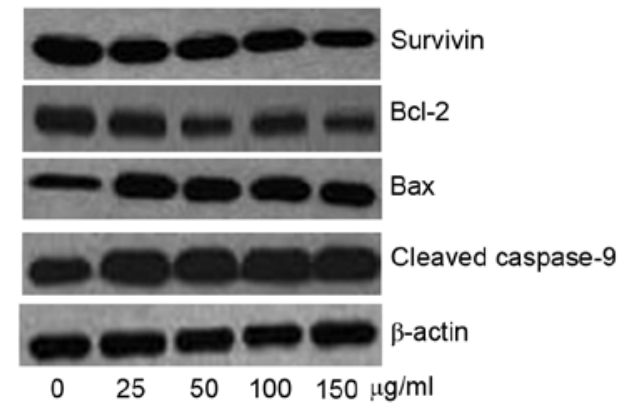

$\mathrm{E}$

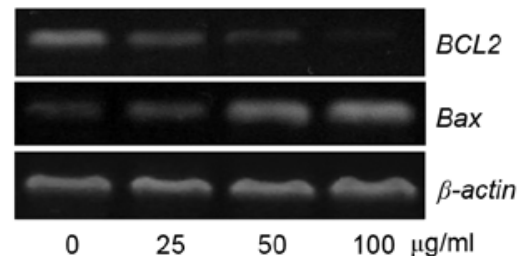

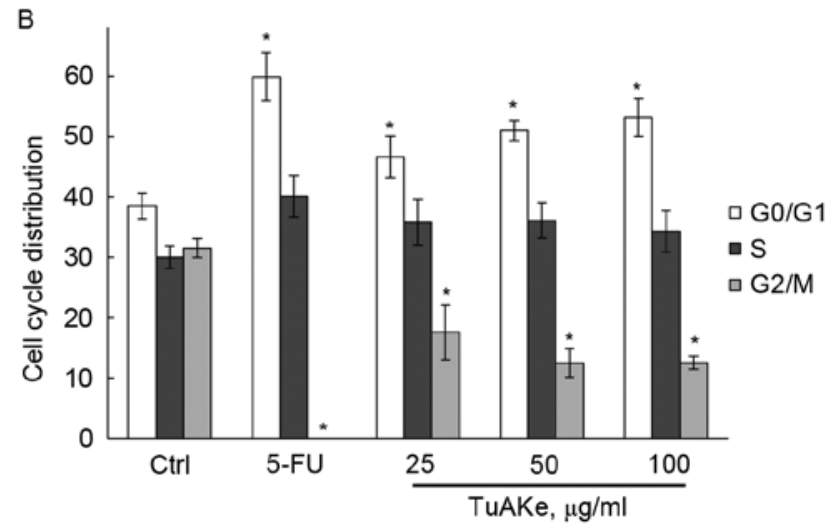

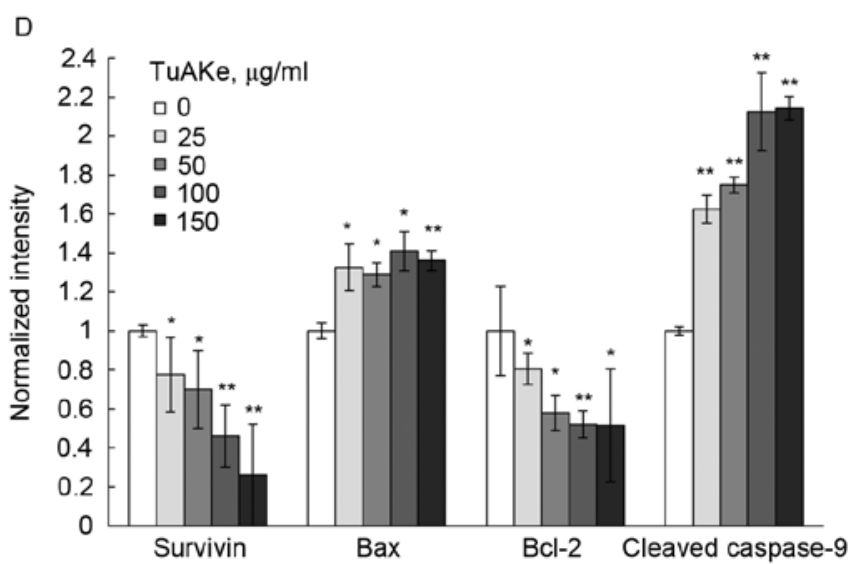

Figure 2. Induction of apoptosis and cell cycle arrest by TuAKe. (A) Cell apoptosis rates and (B) cell cycle distribution by flow cytometry after SGC-7901 cells were treated by different doses TuAKe for $24 \mathrm{~h}$. (C and D) Expression of apoptosis-related proteins survivin, Bax, Bcl-2 and cleaved caspase-9 in SGC-7901 cells treated by TuAKe for $24 \mathrm{~h}$. (C) Representative western blotting images and (D) protein levels normalized to $\beta$-actin (n=3) are shown. (E) Expression of the mRNA levels of $B C L 2, B A X$ and $\beta$-actin by qRT-PCR from SGC-7901 cells treated by TuAKe for $24 \mathrm{~h} .{ }^{*} \mathrm{P}<0.05,{ }^{* *} \mathrm{P}<0.01$ compared with controls (blank).

Cell cycle distribution was analyzed by FCM in SGC-7901 cells treated by TuAKe for $24 \mathrm{~h}$ (Fig. 2B). The percentage of cells in the G0/G1 phase increased from $38.5 \%$ in the control (PBS) group to 46.6-63.9\% in TuAKe-treated groups $(25-100 \mu \mathrm{g} / \mathrm{ml})$; on the other hand, the percentage of cells in the $\mathrm{G} 2 / \mathrm{M}$ phase decreased from $31.5 \%$ in the control group to 12.5-17.6\% in TuAKe-treated groups. Thus, TuAKe treatment resulted in cell cycle arrest in the G0/G1 phase.

TuAKe regulates the expression of apoptosis-associated proteins. To further analyze the molecular evidence of apoptosis, western blottings for several apoptosis-related markers were carried out. Different concentrations of TuAKe could significantly reduce the level of survivin; whereas, there was significant decrease in Bcl-2 as well as increase in Bax and cleaved caspase-9 levels (Fig. 2C and D). By qRT-PCR, it was further demonstrated that TuAKe resulted in decreased $B C L 2$ and increased $B A X$ gene expression (Fig. 2E).

Autophagy induction contributes to TuAKe-induced cell death. Autophagy flux analysis was carried out using HEK293 and AGS cells (Fig. 3A). HEK293, a cell line commonly used in studies on autophagy as a tool, was less sensitive to TuAKe compared with the two gastric cancer cell lines used in this study. The $\mathrm{IC}_{50}$ of TuAKe on HEK293 was higher than the maximum dose tested, $75 \mu \mathrm{g} / \mathrm{ml}$ (Fig. 4B), compared with 35-45 $\mu \mathrm{g} / \mathrm{ml}$ in tumor cells (Fig. 1A). As seen in Fig. 3A, in both HEK293 and AGS cells, TuAKe increased the level of LC3-II, the conjugate of cytosolic autophagy-related protein LC3-I with phosphatidylethanolamine (PE), an indicator of either autophagy activation or blockage of autophagic degradation. In the autophagy flux analysis, CQ, the lysosome inhibitor, increased the level of LC3-II; combination of CQ and TuAKe further increased the level of LC3-II, indicating the effect of TuAKe was to promote the formation of LC3-II instead of blocking its degradation, thus, demonstrating induction of autophagy by TuAKe.

The RFP-GFP-LC3 tag is a well-defined tool to monitor the dynamic formation and degradation of LC3-II-positive autophagosomes. Presence of yellow puncta (RFP-GFP-LC3-II) implies autophagosomes. Moreover, because GFP is degraded faster than RFP in the lysosomes, presence of red puncta (RFP only) implies autolysosomes under degradation at the late stage. In both HEK293 and AGS cells stably expressing RFP-GFPLC3-II, the control cells were free of puncta (Fig. 3B and C), while $\mathrm{TuAKe}$ induced significant increase in the number of yellow puncta. Increase in the number of observable autophagosomes further supported induction of autophagy by TuAKe demonstrated by flux analysis (Fig. 3A).

Observed by EM (Fig. 3D), TuAKe induced significant increase in the number of intracellular membranous organelles 
A
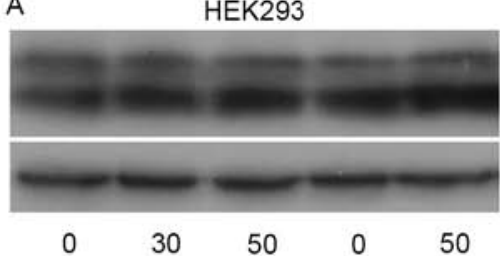

$\begin{array}{lllll}0 & 0 & 0 & 50 & 50\end{array}$

B
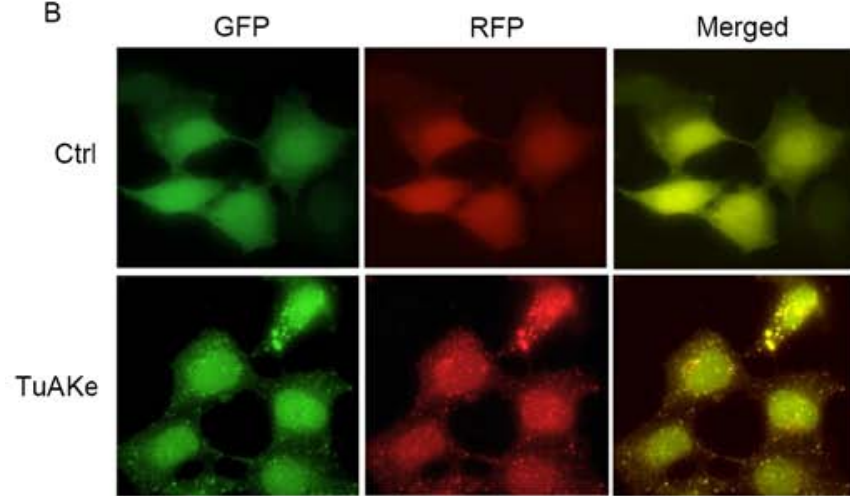

D

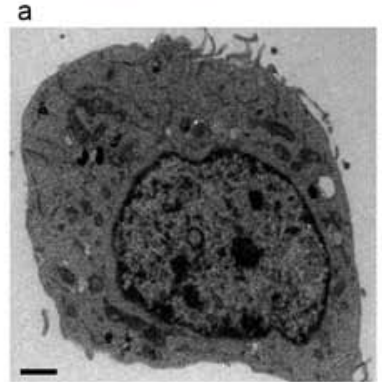

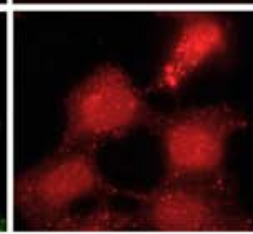

b

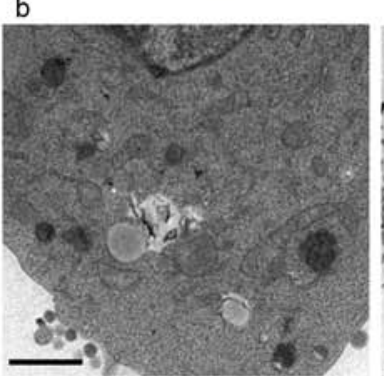

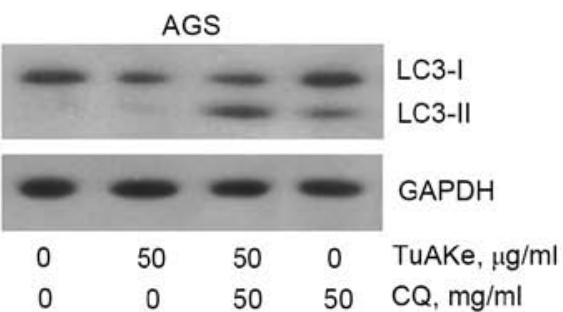

C GFP
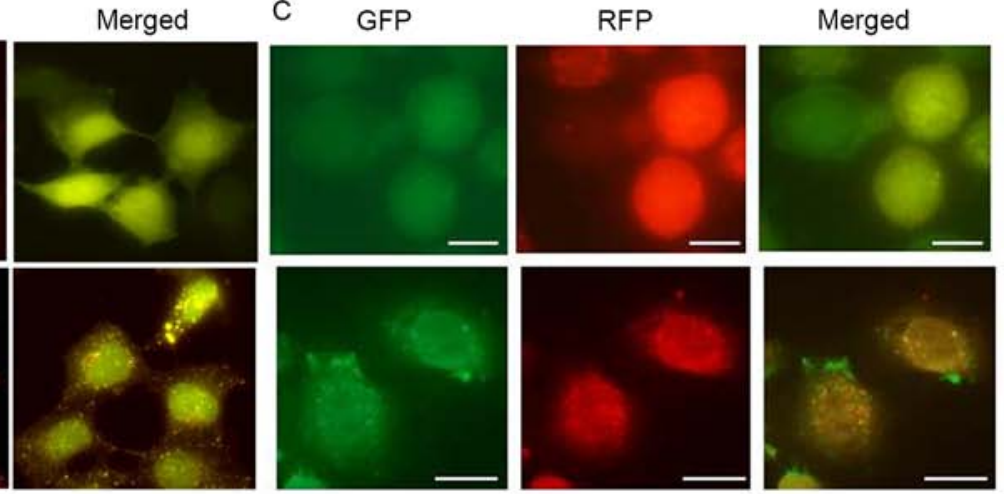

d
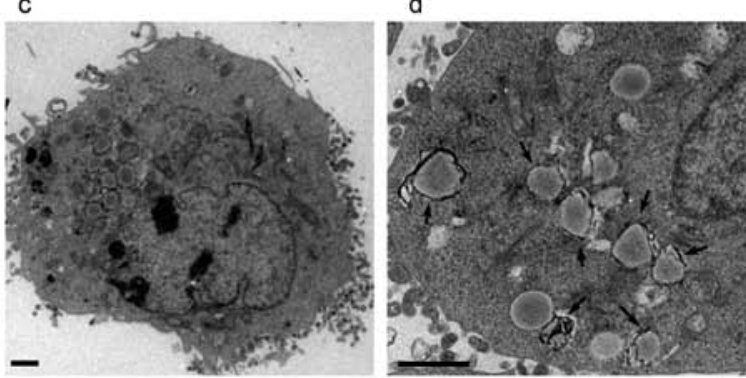

Figure 3. Induction of autophagy by TuAKe. (A) Flux analysis of autophagy. HEK293 and AGS cells were treated by TuAKe for 24 h at the indicated concentrations, or TuAKe combined with chloroquine (CQ) for $6 \mathrm{~h}$ before cells were collected. Cell lysates were analyzed by western blottings to detect LC3 and GAPDH. (B and C) TuAKe-induced autophagosomes under a fluorescence microscope. (B) HEK293 and (C) AGS cells stably expressing RFP-GFP-LC3 were treated by $50 \mu \mathrm{g} / \mathrm{ml}$ TuAKe for $24 \mathrm{~h}$, then fixed and observed under fluorescent microscopy. Scale bar, $20 \mu \mathrm{m}$. (D) TuAKe-induced autophagosomes by electron microscopy. HEK293 cells were treated by PBS (control, Da-b) or by TuAKe at $50 \mu \mathrm{g} / \mathrm{ml}$ for $24 \mathrm{~h}$ (TuAKe, Dc-d). Arrows indicate the presence of autophagosome-like structures. Scale bar, $2 \mu \mathrm{m}$.

Table I. Life quality evaluation of gastric cancer patients after amorphophallus konjac-based therapies.

\begin{tabular}{lcccc}
\hline Group & Improved KPS, $n$ & Stable KPS, $n$ & Decreased KPS, n & Improved or stable KPS, \% (n/n) \\
\hline FAM & 1 & 3 & 6 & $40(4 / 10)$ \\
FAM+TuAK & 10 & 7 & 3 & $85(17 / 20)$ \\
\hline
\end{tabular}

Comparison of the Karnofsky performance status (KPS) scores in patients undergoing chemotherapy with or without amorphophallus konjac tuber (TuAK)-based medicinal decoction. The FAM regimen of chemotherapy was composed of 5-fluorouracil, adriamycin and mitomycin C.

with high electron density, typical of double-membraned autophagosomes (Fig. 3Dc-d). The size of autophagosome-like structures observed under EM was comparable with the LC3-II positive puncta from fluorescent microscopy.

In both HEK293 and AGS cells, after suppression of autophagy by silencing of ATG7 gene, decrease of Atg7 and inhibition of autophagy was confirmed by western blotting (Fig. 4A). After targeted suppression of autophagy, the inhibition rate of TuAKe $(10-75 \mu \mathrm{g} / \mathrm{ml})$ on HEK293 cells was significantly decreased from $5-32 \%$ in the control group to $2-10 \%$ in the siRNA $A T G 7$ group (Fig. 4B). In AGS cells, decrease of inhibition by TuAKe after $A T G 7$ knockdown was only significant when TuAKe was used in higher doses (40-50 $\mu \mathrm{g} / \mathrm{ml})$; however, the inhibition rates between the two groups was highly significant with the net difference of $30-40 \%$.

TuAK improves the life quality of gastric cancer patients. We evaluated the KPS scores in 30 gastric cancer patients, 10 received conventional FAM chemotherapeutic regimen and 20 received FAM regimen plus TuAK-based decoction. As shown in Table I, among the 20 patients in the TuAK group, the KPS 


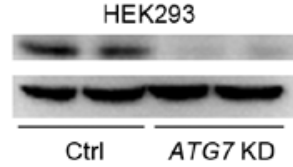

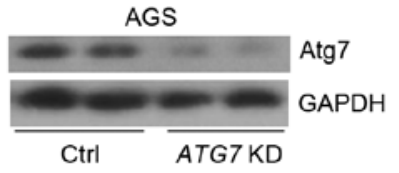

HEK293

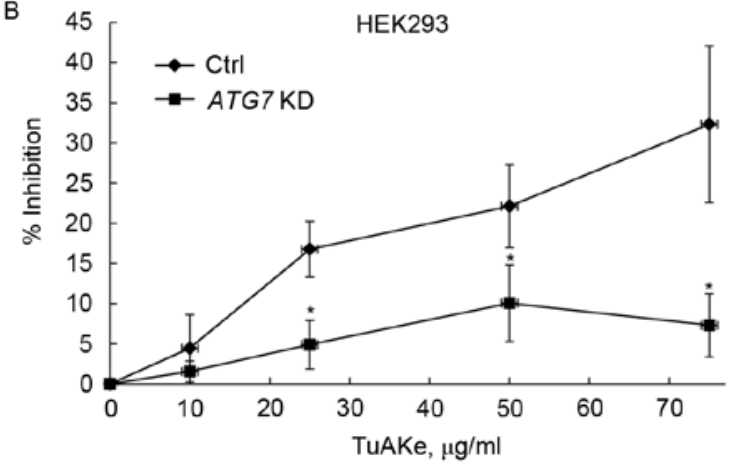

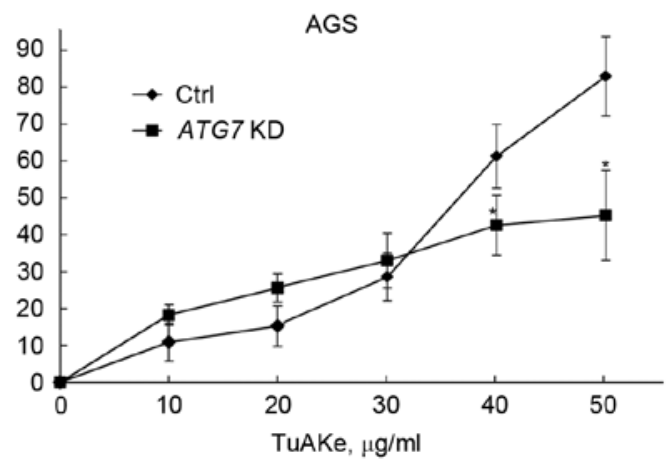

Figure 4. Autophagy is indispensable in TuAKe-induced cytotoxicity. HEK293 and AGS cells with siRNA targeting ATG7 or scrambled siRNA were treated by TuAKe at indicated concentrations for $24 \mathrm{~h}$. (A) Western blotting of Atg7 and GAPDH (internal control) after targeted siRNA. (B) Inhibition rates were calculated after cell viability assay and data are presented as mean $\pm \mathrm{SE}(\mathrm{n}=3) .{ }^{*} \mathrm{P}<0.05$, compared with control siRNA.

scores in 10 had improved, 7 were stable and 3 were worsened; among the 10 patients from the FAM group, 1 had improved KPS score, 3 were stable and 6 were worsened. More patients in the TuAK group obtained improved or stable KPS scores (85\%) compared with those in the FAM only group (40\%), suggesting TuAK-based medication could improve the life quality of gastric cancer patients undergoing chemotherapy.

\section{Discussion}

Despite of its history in the clinical use and the observable beneficial effects in the field of TCM, the tuber part of amorphophallus konjac is not enlisted in the pharmacopeia and its chemical composition is still poorly studied. As a whole plant, Amorphophallus konjac is well tolerated as a food source in some countries. It contains glucomannan $(21,22)$, monosaccharide (23), oligosaccharide (24), polysaccharide (14), aromatic compounds including (+/-)-5,5'-dimethoxysesamin, erythrinasinate, indole-3-carbaldehyde, serotonin, 3,4-dihydroxybenzoic acid, 3,4-dihydroxybenzaldehyde $(13,16)$. The glucomannans are the most studied bioactive components from the powder of amorphophallus konjac, and have been registered in clinical trials in adults $(25)$ and in children $(25,26)$ against obesity and diabetes through regulating food absorption in the gut and subsequently decreasing body weight. Glucomannans are typically extracted by water followed by coagulation by ethanol, which produces much higher yields (27) compared with the organic solvents-based isolation methods used in this study.

Theoretically, aquatic extract contains sugar, amino acids, protein and salts; ethanol could extract most active components besides sugar and proteins; while ligarine extract contains lipids, volatile oil, wax, as well as sterides and terpenes (28). Previous reported information on the chemical composition of the organic extract of TuAK is limited; there is only one report in Chinese on 31 chemicals identified by gas chromatography-mass spectrometry (GC-MS) using extraction method similar as ours (29). In our unpublished data, by high pressure liquid chromatographymass spectrometry (HPLC-MS), up to 71 different chemicals were identified from TuAKe, including nitrogen and sulfurcontaining compounds. From our data and of others, most chemicals contained in the antitumor TuAKe fraction are different species of organic compounds including nitrogen and sulfur-containing compounds. Among the 94 organic compounds identified from whole amorphophallus konjac powder, none was found in the list of toxic compounds by the standards of USFDA (30). From out data, the cytoxicity of TuAKe was significantly lower in non-tumor cell line HEK293 compared with gastric cancer cell lines, supporting the previously reported safety of amorphophallus konjac. It is likely that the TuAKe we obtained does not contain cytotoxic compounds; however, further separation and identification of the chemical compositions are to be carried out in future studies using more sophisticated tools such as NMR.

Our data indicated that the organic solvent-based extract, TuAKe, had much stronger antitumor potency compared with the aqueous extract, suggesting ethanol and ligarine based extraction methods to be more appropriate for the purpose of antitumor treatments of TuAK instead of the traditional water-based extraction. The bioactive chemicals are to be identified. However, according to the holistic theory of TCM, it is also likely that the efficacy is due to the combinational use of multiple ingredients.

We found that TuAKe exhibited antitumor effect at relatively low doses, especially in long-term treatment. Induction of apoptosis and cell cycle distribution reached the saturation point when the concentration of TuAKe was $>50 \mu \mathrm{g} / \mathrm{ml}$. Although safe to consume, our data also suggest that TuAK-based medication in relatively smaller doses would be efficient to achieve desired effects.

A TCM decoction contains hundreds of different chemical components and can modulate many molecular targets simultaneously to result in complicated cascades of cellular events. Qing-Yi-Hua-Ji formula, a TuAK-based decoction, was reported to affect multiple downstream targets including 
Ski (31), Notch 4 and Jagged 1 (32). We have studied how TuAKe regulates cell death pathways. Firstly, TuAKe induced apoptosis by regulation of apoptosis-related proteins including survivin, Bcl-2 and Bax. Survivin belongs to the family of inhibitors of apoptosis proteins (IAP) that regulate cell death by inhibition of caspase activation. It is expressed only in cells in the G2/M phase, but absent in differentiated cells $(33,34)$. Survivin is highly expressed in most human tumors and fetal tissues, and has thus become a biomarker and a therapeutic target in cancer (35). The Bcl-2 family of proteins contain anti-apoptotic proteins including $\mathrm{Bcl}-2$, as well as pro-apoptotic proteins Bax and Bid (36). Decrease of $B C L 2$ and increase of $B A X$ expression was also detected at the mRNA level after TuAKe treatment. Thus, TuAK could regulate apoptosis through the anti-apoptotic IAP and Bcl-2 family proteins, although was not necessarily dependent on them. Moreover, TuAKe could simultaneously increase the levels of the pro-apoptotic proteins Bax and caspase-9. Furthermore, TuAKe was regulatory on cell cycle distribution. It increased the proportion of cells in the G0/G1 phases and decreased those in the G2/M phases. This result is consistent with decrease of survivin, which is expressed in a cell cycle-dependent manner $(33,34)$. Similar regulatory effect of survivin and Bax by TuAKe was also found in hepatocarcinoma cells in our previous study (20).

In the present study, it was found that activation of autophagy was an indispensable antitumor mechanism of TuAKe. By flux analysis, TuAKe was shown to induce autophagy in both HEK293 and AGS cells. By fluorescent imaging of RFP-GFP-LC3 positive puncta, TuAKe was shown to induce the formation of LC3-II-positive puncta representing the maturing autophagosomes again in both cell lines. Under EM, autophagosome-like double membraned structures with high electron densities, a golden marker for autophagy induction, could be induced by TuAKe (37). Judged by the similar scale in size and numbers, the autophagosome-like structures under EM could be the same structure as the RFP-GFP-LC3-II puncta under fluorescent microscopy. To examine whether activation of autophagy contributed to TuAKe-induced cell death, autophagy was specifically inhibited by knocking-down the expression of $A T G 7$, which functions as an E1-like enzyme to form both LC3-II and the Atg5-Atg12 complex in the autophagosome elongation step, and is required for the canonical autophagy $(38,39)$. Suppression of autophagy by knockingdown of $A T G 7$ significantly reduced the anti-proliferative capacity of TuAKe in both HEK293 and AGS cells, implying an essential role of autophagy activation in the biological events induced by TuAKe.

By our retrospective analysis, TuAK-based decoction exhibited beneficial effects in gastric cancer patients undergoing chemotherapy marked by significantly improved or stable KPS scores. The previously mentioned Qing-Yi-Hua-Ji formula, a decoction with TuAK as one of the major ingredients, has also been reported to increase the survival of late stage pancreatic cancer patients (40). However, the decoction is traditionally prepared on water-based extraction, while our data suggest organic solvents could provide extracts with higher antitumor potency.

In conclusion, based on this study, TuAKe inhibits gastric cancer cells through induction of cell cycle arrest, apoptosis, as well as autophagy. Autophagy is indispensable in the antitumor mechanism of TuAKe. Our findings strongly support the use of TuAK-based medication as an alternative or as a combinational component in conventional treatment regimens against gastric cancer, and support the feasibility to isolate active antitumor compounds from TuAK. The detailed bioactive components and the molecular targets are to be revealed by future studies.

\section{Acknowledgements}

The present study is funded by the National Science Foundation of Zhejiang Province, China (no. LY15H160024 to X.C., no. LY16H270006 to L.P.) and by the National Science Foundation of China (no. 81202021 to X.C.).

\section{References}

1. Safarzadeh E, Sandoghchian Shotorbani S and Baradaran B: Herbal medicine as inducers of apoptosis in cancer treatment. Adv Pharm Bull 4 (Suppl 1): 421-427, 2014.

2. Srivastava S, Somasagara RR, Hegde M, Nishana M, Tadi SK, Srivastava M, Choudhary B and Raghavan SC: Quercetin, a natural flavonoid interacts with DNA, arrests cell cycle and causes tumor regression by activating mitochondrial pathway of apoptosis. Sci Rep 6: 24049, 2016.

3. Jiang K, Wang W, Jin X, Wang Z, Ji Z and Meng G: Silibinin, a natural flavonoid, induces autophagy via ROS-dependent mitochondrial dysfunction and loss of ATP involving BNIP3 in human MCF7 breast cancer cells. Oncol Rep 33: 2711-2718, 2015.

4. Kumar G, Mittal S, Sak K and Tuli HS: Molecular mechanisms underlying chemopreventive potential of curcumin: Current challenges and future perspectives. Life Sci 148: 313-328, 2016.

5. Jin $\mathrm{P}$, Zhang $\mathrm{C}$ and $\mathrm{Li} \mathrm{N}$ : Berberine exhibits antitumor effects in human ovarian cancer cells. Anticancer Agents Med Chem 15: 511-516, 2015.

6. Fang LH, Wang RP, Hu SY, Teng YH, Xie WB: The effect of tou nong san on transplanted tumor growth in nude mice. Evid Based Complement Alternat Med 2015: 518454, 2015.

7. Chua M, Baldwin TC, Hocking TJ and Chan K: Traditional uses and potential health benefits of Amorphophallus konjac K. Koch ex N.E.Br. J Ethnopharmacol 128: 268-278, 2010.

8. Committee CP: Pharmacopoeia of the People's Republic of China. 1st edition. China Chemical Industry Press, Beijing, 2010.

9. Luo DY: Inhibitory effect of refined Amorphophallus konjac on MNNG-induced lung cancers in mice. Zhonghua Zhong Liu $\mathrm{Za}$ Zhi 14: 48-50, 1992 (In Chinese).

10. Ansil PN, Nitha A, Prabha SP and Latha MS: Curative effect of Amorphophallus campanulatus (Roxb.) Blume. tuber on $N$-nitrosodiethylamine-induced hepatocellular carcinoma in rats. J Environ Pathol Toxicol Oncol 33: 205-218, 2014.

11. Ansil PN, Wills PJ, Varun R and Latha MS: Cytotoxic and apoptotic activities of Amorphophallus campanulatus tuber extracts against human hepatoma cell line. Res Pharm Sci 9: 269-277, 2014.

12. Ansil PN, Prabha SP, Nitha A and Latha MS: Chemopreventive effect of Amorphophallus campanulatus (Roxb.) blume tuber against aberrant crypt foci and cell proliferation in 1,2-dimethylhydrazine induced colon carcinogenesis. Asian Pac J Cancer Prev 14: 5331-5339, 2013.

13. Mariño G, Niso-Santano M, Baehrecke EH and Kroemer G: Self-consumption: The interplay of autophagy and apoptosis. Nat Rev Mol Cell Biol 15: 81-94, 2014.

14. Booth LA, Tavallai S, Hamed HA, Cruickshanks N and Dent P: The role of cell signalling in the crosstalk between autophagy and apoptosis. Cell Signal 26: 549-555, 2014.

15. White E: Deconvoluting the context-dependent role for autophagy in cancer. Nat Rev Cancer 12: 401-410, 2012.

16. Li T, Tang ZH, Xu WS, Wu GS, Wang YF, Chang LL, Zhu H, Chen XP, Wang YT, Chen Y, et al: Platycodin D triggers autophagy through activation of extracellular signal-regulated kinase in hepatocellular carcinoma HepG2 cells. Eur J Pharmacol 749: 81-88, 2015. 
17. Chen LL, Song JX, Lu JH, Yuan ZW, Liu LF, Durairajan SS and Li M: Corynoxine, a natural autophagy enhancer, promotes the clearance of alpha-synuclein via Akt/mTOR pathway. J Neuroimmune Pharmacol 9: 380-387, 2014.

18. Xu D, Lao Y, Xu N, Hu H, Fu W, Tan H, Gu Y, Song Z, Cao P and $\mathrm{Xu} \mathrm{H}$ : Identification and characterization of anticancer compounds targeting apoptosis and autophagy from Chinese native Garcinia species. Planta Med 81: 79-89, 2015.

19. Chen PF and Liu LM: Observation on the anti-tumor effects and induction carcinoma cell's apoptosis of She Liu Gu. Chinese J Bas Med TCM 6: 30-32, 2000 (In Chinese).

20. Pan L and Chen PF: In vitro and in vivo suppression of hepatocellular carcinoma by amorphophallus konjac tuber through regulation of survivin and bax. Oncol Lett (In press).

21. Maiuri MC, Zalckvar E, Kimchi A and Kroemer G: Self-eating and self-killing: Crosstalk between autophagy and apoptosis. Nat Rev Mol Cell Biol 8: 741-752, 2007.

22. Li B, Xia J, Wang Y and Xie B: Grain-size effect on the structure and antiobesity activity of konjac flour. J Agric Food Chem 53: 7404-7407, 2005.

23. Fan YJ and Zong WX: The cellular decision between apoptosis and autophagy. Chin J Cancer 32: 121-129, 2013.

24. Rubinstein AD and Kimchi A: Life in the balance - a mechanistic view of the crosstalk between autophagy and apoptosis. J Cell Sci 125: 5259-5268, 2012.

25. Zalewski BM, Chmielewska A, Szajewska H: The effect of glucomannan on body weight in overweight or obese children and adults: A systematic review of randomized controlled trials. Nutrition 31: 437-442e432, 2015.

26. Zalewski BM and Szajewska H: Effect of glucomannan supplementation on body weight in overweight and obese children: Protocol of a randomised controlled trial. BMJ Open 5: e007244, 2015.

27. Harmayani E, Aprilia V and Marsono Y: Characterization of glucomannan from Amorphophallus oncophyllus and its prebiotic activity in vivo. Carbohydr Polym 112: 475-479, 2014.

28. XH K: Chemistry of Chinese Materia Medica. China Press of Traditional Chinese Medicine, Beijing, 2003.
29. Li JHX: Study on Chemical Components of Petroleum Ether Fraction of Alcohol Extract Obtained from Amorphophallus Blume. J Xihua University-Natural Sci 28: 68-69, 2009.

30. Jy Y: The cultivation and application of Amorphophallus Konjac. Hangzhou University Press, Hangzhou, 1991.

31. Wang P, Chen Z, Meng ZQ, Luo JM, Lin JH, Zhou ZH, Chen H, Wang K, Shen YH and Liu LM: Ski acts as therapeutic target of qingyihuaji formula in the treatment of SW1990 pancreatic cancer. Integr Cancer Ther 9: 50-58, 2010.

32. Xu Y, Zhu F, Xu S and Liu L: Anti-tumor effect of the extract from qingyihuaji formula on pancreatic cancer by down-regulating Notch-4 and Jagged-1. J Tradit Chin Med 35: 77-83, 2015.

33. Altieri DC: Validating survivin as a cancer therapeutic target. Nat Rev Cancer 3: 46-54, 2003.

34. Sah NK, Khan Z, Khan GJ and Bisen PS: Structural, functional and therapeutic biology of survivin. Cancer Lett 244: 164-171, 2006.

35. Altieri DC: Survivin, cancer networks and pathway-directed drug discovery. Nat Rev Cancer 8: 61-70, 2008.

36. Chao DT and Korsmeyer SJ: BCL-2 family: Regulators of cell death. Annu Rev Immunol 16: 395-419, 1998.

37. Mizushima N, Yoshimori $\mathrm{T}$ and Levine B: Methods in mammalian autophagy research. Cell 140: 313-326, 2010.

38. Komatsu M, Waguri S, Ueno T, Iwata J, Murata S, Tanida I, Ezaki J, Mizushima N, Ohsumi Y, Uchiyama Y, et al: Impairment of starvation-induced and constitutive autophagy in Atg7deficient mice. J Cell Biol 169: 425-434, 2005.

39. Nakatogawa $H$ : Two ubiquitin-like conjugation systems that mediate membrane formation during autophagy. Essays Biochem 55: 39-50, 2013.

40. Liu LWL and Lin S: Therapeutic evaluation on advanced pancreatic cancer treated by integrative Chinese and western medicine: Clinical analysis of 56 cases. Chin J Integr Med 10: 236-237, 2004. 